15

\title{
Сканирующий оптический квантовый магнитометр, основанный на явлении выжигания провалов
}

\author{
() А.Н. Анисимов, Р.А. Бабунц, И.Д. Бреев, А.П. Бундакова, И.В. Ильин, М.В. Музафарова, П.Г. Баранов \\ Физико-технический институт им. А.Ф. Иоффре РАН, Санкт-Петербург, Россия \\ ๑ E-mail: pavel.baranov@mail.ioffe.ru
}

Поступило в Редакцию 24 января 2019г.

В окончательной редакции 20 февраля 2019 г.

Принято к публикации 20 фревраля 2019 г.

\begin{abstract}
Предложен сканирующий оптический квантовый магнитометр с субмикронным пространственным разрешением, основанный на физическом явлении выжигания провалов в сигнале оптически детектируемого магнитного резонанса (ОДМР) на спиновых центрах окраски атомарных размеров в карбиде кремния в условиях насыщения спиновых уровней дополнительной высокочастотной резонансной накачкой. Увеличение чувствительности обусловлено сужением линии ОДМР и увеличением крутизны зависимости частоты сигнала от магнитного поля.
\end{abstract}

DOI: 10.21883/PJTF.2019.10.47751.17710

Обнаружение слабых магнитных полей является важнейшей проблемой в различных областях как в фундаментальной физике, так и в прикладных исследованиях. Особую роль в магнитометрии играют оптические методы измерения магнитных полей, основанные на спиновых свойствах паров щелочных элементов с использованием ячеек миллиметровых размеров, которые позволяют измерять магнитные поля с чрезвычайно высокой чувствительностью $[1,2]$. В последнее десятилетие взрывное развитие получили оптические квантовые магнитометры, основанные на уникальных свойствах спиновых центров окраски атомарных размеров в кристаллах алмаза [3] и карбида кремния (SiC) [4-10], что может обеспечить измерение распределения магнитных полей с микронным и субмикронным разрешением. Оптическое облучение центров окраски приводит к выстраиванию населенностей спиновых уровней, а возбуждение резонансных переходов между спиновыми уровнями вызывает значительные изменения интенсивности фотолюминесценции (ФЛ) этих центров. Оптическое детектирование магнитного резонанса наблюдается в широком диапазоне температур, вплоть до комнатной температуры и выше, при этом появляется возможность регистрации одиночных спинов $[3,7,10]$. Принцип магнитометрии основан на зависимости сигнала оптически детектируемого магнитного резонанса (ОДМР) от измеряемого внешнего магнитного поля в соответствии с зеемановским расщеплением спиновых уровней. Оптически адресуемый, управляемый микроволнами и сохраняющий квантовую когерентность даже при комнатной температуре электронный спин центра окраски в $\mathrm{SiC}$ можно использовать в качестве магнитного датчика. Преимущество сканирующего магнитометра на основе спиновых центров окраски заключается в разнообразных вариантах устройств: 1) ансамблевые спиновые центры окраски, расположенные близко к поверхности $\mathrm{SiC}$, на расстоянии нескольких нанометров (с возможностью со- здавать заданное распределение центров окраски в кристалле $\mathrm{SiC}$ [11]), могут обнаруживать малые магнитные поля магнитных структур, электронные и ядерные спины различных материалов, помещенных на поверхности $\mathrm{SiC}$, что обеспечивает субмикронное масштабирование и двумерную магнитную визуализацию; 2) при помещении спиновых центров в сканирующий зонд на основе $\mathrm{SiC}$ достижимо пространственное разрешение в атомном масштабе; 3) флуоресцентные нанокристаллы $\mathrm{SiC}$, coдержащие спиновые центры окраски, могут рассматриваться как функциональные устройства атомарных масштабов для измерения магнитных и температурных полей.

На рис. 1 показана блок-схема сканирующего оптического квантового магнитометра. В него входит лазер накачки, разветвитель луча и объектив, активный материал в виде кристалла $\mathrm{SiC}$, содержащего спиновые центры окраски (их структура приведена слева), расположенного на сканирующем столике конфокального микроскопа, на который помещается исследуемый образец, а также фотодетектор, синхронный детектор, низкочастотный (НЧ) генератор, зондирующий высокочастотный (ВЧ) генератор с частотой $f$ (probe), ВЧ-генератор накачки с частотой $f(p)$, катушка электромагнита, на которую подается постоянный или низкочастотный переменный ток. Рассматриваемый спиновый центр окраски в $\mathrm{SiC}$ представляет собой комбинацию вакансий кремния и углерода, характеризующуюся общим спином $S=3 / 2$, ось симметрии спинового центра совпадает с осью $c$ кристалла, условная структура приведена на рис. 1. При оптическом возбуждении в ближнем ИК-диапазоне $(750-850 \mathrm{~nm})$ осуществляется выстраивание спинов центров окраски, при этом создается неравновесное заполнение спиновых уровней (верхняя вставка на рис. 1). Изменение заполнения спиновых уровней путем облучения кристалла ВЧ-излучением с частотой магнитного резонанса приводит к изменению интенсивности ФЛ спиновых центров 


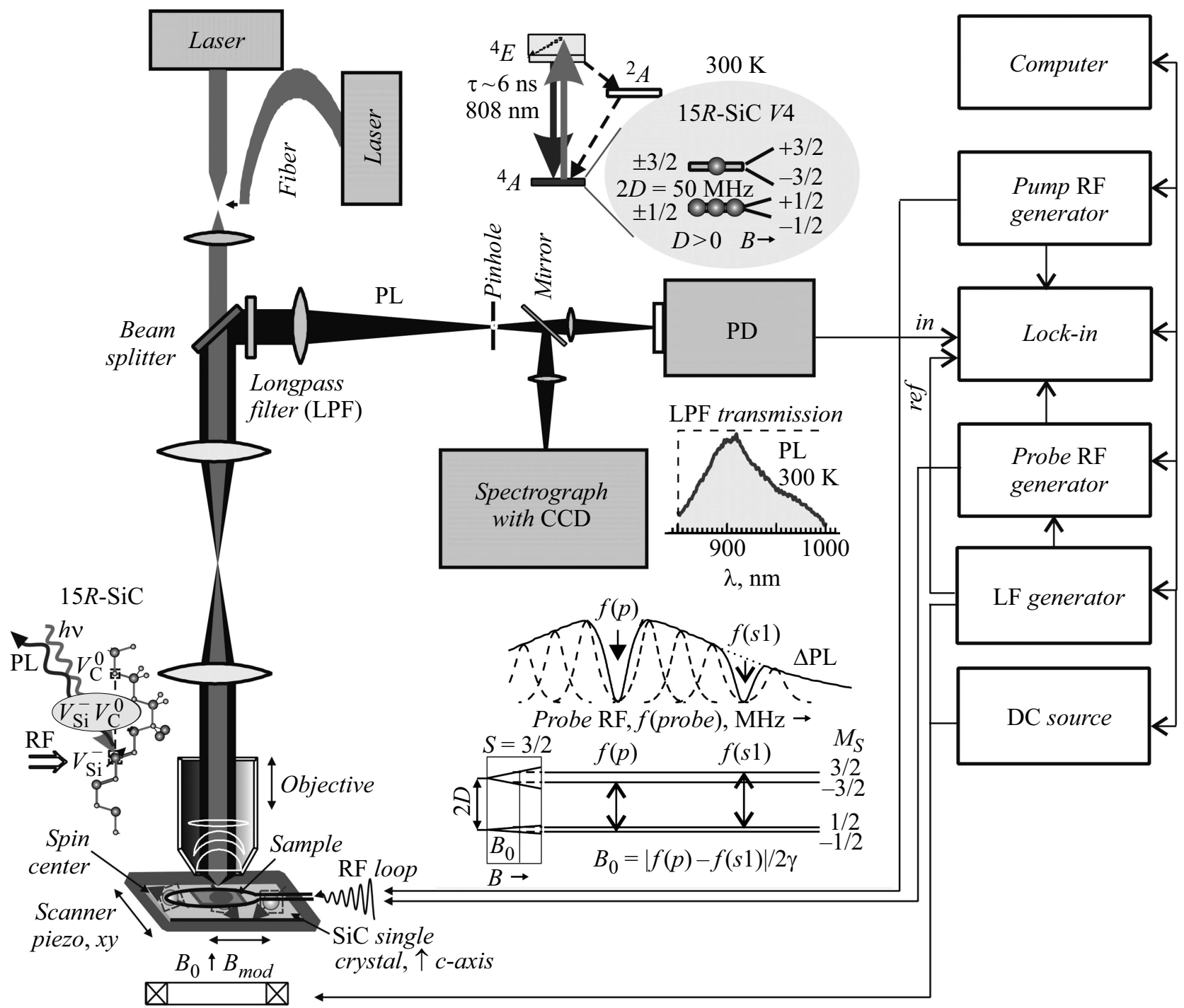

Рис. 1. Блок-схема сканирующего оптического квантового магнитометра, схема оптического выстраивания спинов центра $V 4$ в кристалле $15 R-\mathrm{SiC}$, спектр ФЛ и условная схема, поясняющая механизм выжигания провалов в неоднородно уширенной линии ОДМР.

(в диапазоне 850-950 nm, рис. 1) в момент магнитного резонанса.

Спиновый гамильтониан $H$, описывающий спиновые подуровни для $S=3 / 2$ в магнитном поле $\mathbf{B}$, ориентированном вдоль гексагональной оси $c(z)$, имеет вид

$$
H=g \mu_{\mathrm{B}} \mathbf{S}_{z} \mathbf{B}+D\left[\mathbf{S}_{z}^{2}-1 / 3 S(S+1)\right]
$$

где первое и второе слагаемые отражают зеемановское расщепление спиновых уровней в магнитном поле и расщепление тонкой структуры в аксиальном поле соответственно. Произведение электронного $g$-фактора спинового центра на магнетон Бора $\mu_{\mathrm{B}}$ является гиромагнитным отношением $\gamma=g \mu_{\mathrm{B}} ; D-$ параметр тонкой структуры, для центра с $S=3 / 2$ расщепление тонкой структуры в нулевом магнитном поле $\Delta v=2|D|$ (параметры $D$ приведены в [10]).
Частоты переходов между уровнями с разными проекциями спина $M_{S}$ на направление внешнего магнитного поля, ориентированного вдоль аксиальной оси спинового центра, находятся по формулам

$$
\begin{gathered}
f(-3 / 2 \leftrightarrow-1 / 2)=2 D / h-\gamma B, \\
f(3 / 2 \leftrightarrow 1 / 2)=2 D / h+\gamma B,
\end{gathered}
$$

$h$ - постоянная Планка. Отметим, что аксиальные оси всего ансамбля спиновых центров с квадруплетным основным состоянием в кристалле $\mathrm{SiC}$ совпадают, т.е. частота перехода (зеемановская частота) зависит линейно от магнитного поля $B$ с коэффициентом пропорциональности $\gamma$.

Чувствительность магнитометра на основе ОДМР на активных спиновых центрах ограничена шириной линии ОДМР и крутизной зависимости зеемановской частоты 
от магнитного поля. Задачей настоящей работы является разработка оптического квантового магнитометра, использующего изменение интенсивности ФЛ в момент магнитного резонанса, в котором чувствительность повышается вследствие уменышения ширины линии ОДМР и увеличения коэффициента пропорциональности между частотой ОДМР и магнитным полем. Для решения поставленной задачи используется методика „выжигания провалов“" (ВП), „hole-burning“, с помощью дополнительной ВЧ-накачки на постоянной частоте, как это применялось ранее в фотонике [12].

Основной причиной большой ширины линии ОДМР является неоднородное уширение, которое обусловлено локальными неоднородностями напряжений и магнитных полей. На нижней вставке к рис. 1 условно представлена неоднородно уширенная линия ОДМР, состоящая из однородно уширенных компонент (штриховые линии). В ней показаны выжженные провалы под действием ВЧ-накачки на частоте накачки $f(p)$ и на частоте $f(s 1)$ сателлита, возникающего из-за наличия малого магнитного поля, для спиновых центров с определенным расщеплением тонкой структуры (определенным параметром $D$ спинового гамильтониана). Внизу показаны структура энергетических спиновых уровней и соответствующие частоты $f(p)$ и $f(s 1)$. ВЧ-накачка насыщает спиновый переход $M_{S}=-1 / 2 \leftrightarrow M_{S}=-3 / 2$ с одним конкретным $D$. Из-за спиновой релаксации он воздействует на переход $M_{S}=+1 / 2 \leftrightarrow M_{S}=+3 / 2$ с тем же $D$. Следовательно, сателлит ВП должен появиться при $f(s 1)=f(p)+2 \gamma B$. Если неоднородное уширение больше зеемановского расщепления, та же накачка также насыщает переход $M_{S}=+1 / 2 \leftrightarrow M_{S}=+3 / 2$, но с другим $D$. Соответственно он влияет на переход $M_{S}=-1 / 2 \leftrightarrow M_{S}=-3 / 2$, а другой сателлит появляется при $f(s 2)=f(p)-2 \gamma B$. Таким образом, коэффициент для линейной зависимости от магнитного поля равен $2 \gamma$, т. е. в 2 раза выше, чем при использовании стандартного ОДМР на одном резонансном переходе, где соответствующий коэффициент равен гиромагнитному отношению $\gamma$ (использование двух резонансных переходов для измерения магнитного поля затруднительно изза перекрытия сигналов ОДМР вследствие большой ширины линий). Оба сателлита могут использоваться для измерения магнитного поля, причем расстояние между ними $|f(s 1)-f(s 2)|=4 \gamma B$, т. е. коэффициент для линейной зависимости от магнитного поля повышается еще в 2 раза и равен $4 \gamma$. Для измерения магнитного поля определяются частоты сателлитов, зависящие от расщепления зеемановских уровней в магнитном поле. Затем магнитное поле, в котором находится область кристалла, возбуждаемая сфокусированным лазерным светом, рассчитывается по разнице частот.

Блок-схема магнитометра представлена на рис. 1. Оптический магнитометр включает лазер накачки, излучающий свет в ИК-диапазоне (например, 795 или $808 \mathrm{~nm}$ ), который через разветвитель луча в виде полупрозрачного зеркала и объектива фокусирует лазер- ный луч на активный материал, представляющий собой кристалл карбида кремния, содержащий спиновые центры окраски, при этом выделяется возбуждаемый лазером объем активного материала, в котором измеряется магнитное поле. ФЛ активных спиновых центров через ту же оптическую систему в виде объектива и полупрозрачного зеркала, а также фильтра, отсекающего возбуждающий свет, поступает на фотодетектор, выполненный, например, в виде ФЭУ, фотодиода, лавинного фотодиода, и далее на синхронный детектор, на который также подается опорная частота от НЧ-генератора. На НЧ осуществляются различные опции по модуляции ВЧ-генераторов, ВЧ-излучение которых через систему подачи ВЧ-мощности в виде витка или катушки индуцирует переходы магнитного резонанса между спиновыми уровнями активных спиновых центров в оптически выделенном сфокусированным светом объеме активного материала. ВЧ-мощность подается от двух ВЧ-генераторов: зондирующего ВЧ-генератора (probe radiofrequency (RF) generator), частота которого $f$ (probe) может изменяться в пределах линии ОДМР, и ВЧ-генератора накачки (pump RF generator) с постоянной частотой $f(p)$, находящейся в пределах линии ОДМР. Генератор накачки используется для выжигания узких провалов в линии ОДМР на частоте накачки $f(p)$ и частотах сателлитов $f(s)$, возникающих при расщеплении спиновых уровней во внешнем магнитном поле. Величина измеряемого магнитного поля определяется по разности частот $f(p)$ и $f(s)$ с использованием формулы для зеемановского расщепления в соответствии со спиновым гамильтонианом (1). Предлагается применение четырех различных режимов работы, использующих НЧгенератор для модуляции и синхронное детектирование изменения ФЛ в зависимости от частоты зондирующего ВЧ-генератора: I - НЧ-амплитудная модуляция мощности ВЧ-генератора накачки; II - НЧ-амплитудная модуляция мощности ВЧ-генератора зондирования; III - НЧ-модуляция частоты ВЧ-генератора накачки; IV - НЧ-модуляция частоты ВЧ-генератора зондирования. Подача постоянного и модуляционного магнитных полей осуществляется с применением дополнительной катушки от источника постоянного тока и НЧ-генератора. Используется сканирующий конфокальный оптический микроскоп, в котором осуществляется возвратно-поступательное перемещение в двух взаимно перпендикулярных направлениях под действием управляющих напряжений стола с пьезоэлементом, на котором расположен активный материал в виде пластины кристалла $\mathrm{SiC}$ со спиновыми центрами. Сканирование по третьему направлению осуществляется путем возвратно-поступательного перемещения объектива. Возможна также инвертированная конструкция конфокального микроскопа компании НТ МДТ, в которой объектив конфокального микроскопа (используются объективы с числовой апертурой NA от 1.4 до 0.6) расположен под сканирующим столиком. Всеми процессами магнитометра управляет компьютер. Для 


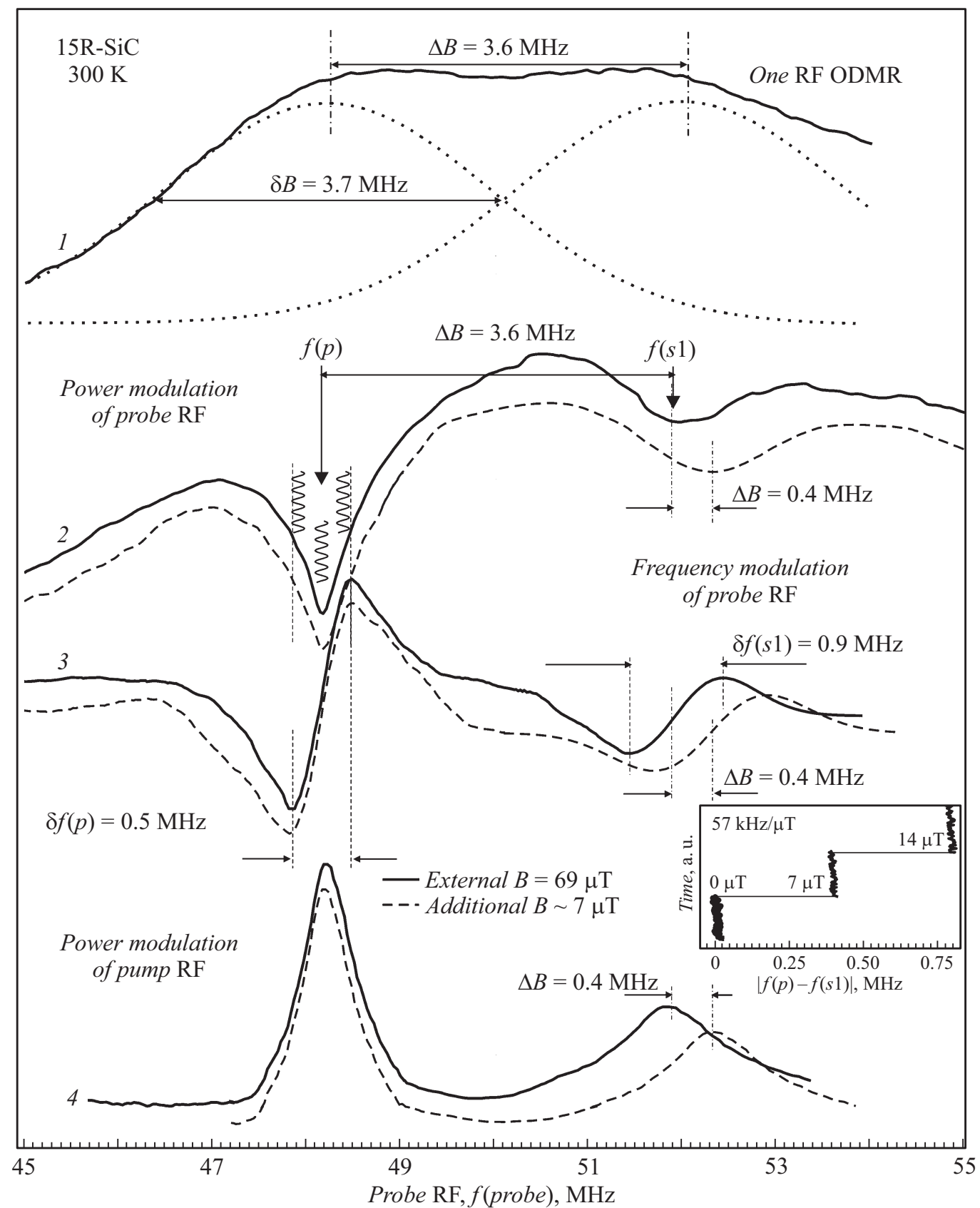

Рис. 2. Спектры ОДМР, зарегистрированные по изменению ФЛ спиновых центров окраски в кристалле $15 R-\mathrm{SiC}$ с использованием синхронного детектирования (вертикальными волнистыми линиями условно показана модуляция частоты) в объеме возбуждения ФЛ. 1 - при НЧ-амплитудной модуляции мощности ВЧ-генератора зондирования, 2 - при НЧ-амплитудной модуляции мощности ВЧ-генератора зондирования при приложении второй ВЧ-накачки $f(p), 3$ - при НЧ-модуляции частоты ВЧ-генератора зондирования при приложении второй ВЧ-накачки $f(p), 4$ - при НЧ-модуляции мощности ВЧ-генератора накачки с приложением частоты зондирования $f$ (probe). Штриховыми линиями показаны спектры, зарегистрированные в дополнительном внешнем магнитном поле $7 \mu \mathrm{T}$. На вставке - разности частот $|f(p)-f(s 1)|$, которые используются для определения локального магнитного поля в объеме кристалла, возбуждаемого сфокусированным лазерным лучом, при ступенчатом изменении внешнего магнитного поля от 0 до 7 и $14 \mu \mathrm{T}$.

измерения магнитных полей в различных исследуемых объектах (образцах) последние помещаются на поверхность активного материала в виде кристалла карбида кремния со спиновыми центрами окраски, в котором возбуждается ФЛ и регистрируется ОДМР. В результате характеристики сигналов ОДМР зависят от измеряемых магнитных полей в исследуемом образце.

Для демонстрации работы магнитометра выбран активный материал в виде кристалла $15 R-\mathrm{SiC}$ со спиновыми центрами $V 4$, расщепление тонкой структуры ко- 
торых $2 D=50.2 \mathrm{MHz}$. На рис. 2 показана неоднородно уширенная линия ОДМР, зарегистрированная в кристалле $15 R$-SiC при комнатной температуре с нескомпенсированным магнитным полем $69 \mu$ Т (видно расщепление линии на две компоненты) в точке возбуждения люминесценции без подачи мощности ВЧ-генератора накачки (1). На рис. 2 спектры 2, 3 и 4 зарегистрированы с использованием трех из четырех предложенных выше режимов работы (I, II и III). Регистрация осуществлялась с использованием синхронного детектирования на низкой частоте $330 \mathrm{~Hz}$. Использовалась частота накачки $f(p)=48.14 \mathrm{MHz}$. В области $f(p)$ наблюдается узкий выжигаемый провал, справа виден выжигаемый провал на частоте сателлита $f(s 1)$. Штриховыми линиями показаны соответствующие спектры, зарегистрированные в дополнительном внешнем поле $7 \mu$ Т. Полезно ввести характеристику, отражающую относительную чувствительность в определении магнитного поля по отношению сдвига линии в одном и том же магнитном поле $(\Delta B)$ к ширине линии $(\delta B): \Delta B / \delta B$ (рис. 2). Видно, что для спектра $1 \Delta B / \delta B \cong 1$, тогда как в случае ВП $\Delta B / \delta B \cong 4$ (спектры $2-4)$.

На вставке к рис. 2 показана разность частот $|f(p)-f(s 1)|$, которая используется для определения локального магнитного поля в объеме кристалла, возбуждаемого сфокусированным лазерным лучом, при ступенчатом изменении внешнего магнитного поля от 0 до 7 и $14 \mu \mathrm{T}$. Данные находятся в соответствии с формулами для вычисления магнитного поля: $B=|f(p)-f(s 1)| / 2 \gamma$. Время записи полного спектра составляло $\sim 10 \mathrm{~s}$, а время записи фрагментов спектра, необходимых для измерения магнитного поля, составляло менее $1 \mathrm{~s}$. Чувствительность такого измерения исходя из величины шума, который приводит к неопределенности определения частоты $\sim 0.1 \mathrm{MHz}$, согласно формуле $B=|f(p)-f(s 1)| / 2 \gamma$, соответствует чувствительности в определении изменения магнитного поля $\sim 1 \mu \mathrm{T} / \sqrt{\mathrm{Hz}}$.

Если оптимизировать регистрирующую систему, может быть достигнута чувствительность $\sim 100 \mathrm{nT} / \sqrt{\mathrm{Hz}}$ в пределах объема порядка $10^{-9} \mathrm{~mm}^{3}$, ограниченного дифракционным пределом, а при использовании нелинейных свойств флуоресценции образцов - микроскопии на основе подавления спонтанного испускания (stimulated emission depletion microscopy) - оптически возбуждаемый и излучаемый объем может быть ограничен вплоть до нескольких нанометров. Дальнейшее повышение чувствительности возможно при использовании импульсных методов ОДМР и выборе оптимального материала карбида кремния с пониженной концентрацией парамагнитных дефектов и обедненным содержанием изотопов ${ }^{29} \mathrm{Si}$ и ${ }^{13} \mathrm{C}$ с ядерными магнитными моментами, как это ранее обсуждалось для $\mathrm{N} V$-центров в алмазе [13].

\section{Финансирование работы}

Работа выполнена при поддержке Министерства науки и высшего образования РФ (соглашение
№ 14.604.21.0200, идентификатор проекта RFMEFI60417X0200).

\section{Список литературы}

[1] Budker D., Romalis M.V. // Nature Phys. 2007. V. 3. P. $227-$ 234.

[2] Александров Е.Б., Вершовский А.К. // УФН. 2009. Т. 179. № 6. C. 605-637.

[3] Gruber A., Drabenstedt A., Tietz C., Fleury L., Wrachtrup J., von Borczyskowski C. // Science. 1997. V. 276. P. 2012-2014.

[4] Baranov P.G., Bundakova A.P., Soltamova A.A., Orlinskii S.B., Borovykh I.V., Zondervan R., Verberk R., Schmidt J. // Phys. Rev. B. 2011. V. 83. P. 125203.

[5] Kraus H., Soltamov V.A., Riedel D., Väth S., Fuchs F., Sperlich A., Baranov P.G., Dyakonov V., Astakhov G.V. // Nature Phys. 2014. V. 10. P. 157-162.

[6] Simin D., Fuchs F., Sperlich A., Baranov P.G., Astakhov G.V., Dyakonov V. // Phys. Rev. Appl. 2015. V. 4. P. 014009.

[7] Widmann M., Lee S.Y., Rendler T., Son N.T., Fedder H., Paik S., Yang L.P., Zhao N., Yang S., Booker I., Denisenko A., Jamali M., Momenzadeh S.A., Gerhardt I., Ohshima T., Gali A., Janzén E., Wrachtrup J. // Nature Mater. 2015. V. 14. P. 164-168.

[8] Simin D., Soltamov V.A., Poshakinskiy A.V., Anisimov A.N., Babunts R.A., Tolmachev D.O., Mokhov E.N., Trupke M., Tarasenko S.A., Sperlich A. // Phys. Rev. X. 2016. V. 6. P. 031014.

[9] Анисимов А.Н., Толмачев Д.О., Бабуни, Р.А., Музабарова М.В., Бундакова А.П., Ильин И.В., Солтамов В.А., Баранов П.Г., Мохов E.H., Astakhov G.V., Dyakonov V. // Письма в ЖТФ. 2016. Т. 42. В. 12. С. 22-29.

[10] Baranov P.G., von Bardeleben H.J., Jelezko F., Wrachtrup J. Magnetic resonance of semiconductors and their nanostructures: basic and advanced applications. Springer Ser. in Materials Science. V. 253. Wien: Springer-Verlag, 2017. $524 \mathrm{p}$.

[11] Ohshima T., Satoh T., Kraus H., Astakhov G.V., Dyakonov V., Baranov P.G. // J. Phys. D: Appl. Phys. 2018. V. 51. P. 333002.

[12] Persistent spectral hole-burning: science and applications / Ed. W.E. Moerner. Topics in Current Physics. V. 44. BerlinHeidelberg: Springer, 1988. $315 \mathrm{p}$.

[13] Balasubramanian G., Chan I.Y., Kolesov R., Al-Hmoud M., Tisler J., Shin C., Kim C., Wojcik A., Hemmer P.R., Krueger A., Hanke T., Leitenstorfer A., Bratschitsch R., Jelezko F., Wrachtrup J. // Nature. 2008. V. 455. P. 648-651. 